\title{
The Influence of Sex on the Sleep-Cardiovascular Disease Relationship: a Review
}

\author{
Zarina Nestor ${ }^{1} \cdot$ Sapna Siddharth $^{2} \cdot$ Ridhwan Y. Baba $^{3}$. \\ Neomi Shah ${ }^{4,5,6}$
}

Published online: 28 September 2015

(C) Springer International Publishing AG 2015

\begin{abstract}
Obstructive sleep apnea (OSA) is a chronic condition characterized by frequent episodes of upper airway collapse during sleep that affects nocturnal sleep quality and often manifests as symptoms of daytime fatigue and sleepiness. Traditionally considered to be a condition uncommon in the female population, recent large population-based epidemiological studies have reported a much higher prevalence of OSA in females than previously estimated. This shift has brought attention to the influence of sex on risk factors and various health-related consequences associated with OSA. Specifically, there is increasing interest in the sex-related differences in cardiovascular outcomes of OSA - such as hypertension, coronary artery disease, stroke, and related mortality. Although the mechanisms underlying the sex-related differences in OSA itself have been explored, the influence of sex on the relationship between OSA and cardiovascular disease
\end{abstract}

This article is part of the Topical Collection on Cardiovascular Disease

Neomi Shah

nshah@montefiore.org; neomi.shah@einstein.yu.edu

1 Department of Medicine, Montefiore Medical Center, 111 East 210th Street, Bronx, NY 10467, USA

2 Department of Medicine, Montefiore Medical Center Wakefield Campus, 600 East 233rd Street, Bronx, NY 10466, USA

3 Department of Medicine, Case-Western Reserve University, Cleveland, OH 44109, USA

4 Department of Medicine, Montefiore Medical Center, 3411 Wayne Avenue, Bronx, NY 10467, USA

5 Department of Epidemiology \& Population Health, Albert Einstein College of Medicine, Bronx, NY 10467, USA

6 Department of Medicine, Albert Einstein College of Medicine, Montefiore Medical Center, 111 East 210th Street, Bronx, NY 10467, USA has not been adequately examined. Our review assesses this influence of sex on the relationship between OSA and cardiovascular disease. We emphasize the need for further research to improve our understanding of the effect of sex on the relationship between OSA and cardiovascular disease.

Keywords Sleep apnea $\cdot$ Obstructive sleep apnea $\cdot$ Coronary heart disease $\cdot$ Cardiovascular disease $\cdot$ Sex differences . Cardiovascular outcomes $\cdot$ Heart failure $\cdot$ Chronic heart failure $\cdot$ Hypertension $\cdot$ Stroke

\section{Introduction}

Obstructive sleep apnea (OSA) is a highly prevalent sleep disorder that is associated with significant morbidity and mortality. Using a polysomnography derived definition of $\mathrm{AHI} \geq$ 5 , the prevalence of OSA accompanying daytime sleepiness is approximately $3-7 \%$ in adult men and $2-5 \%$ in adult women amongst the general population $[1 \bullet \bullet]$. Although initially considered a condition uncommon in the female population, several recent large epidemiological studies of the general population have reported a male to female prevalence ratio of approximately 2 to $3: 1$, much lower than was previously estimated $[2,3]$.

Further, the consequences of sleep disorders, especially OSA, have been of special interest over the last two decades. Recent data confirm an association between OSA and cardiovascular disease; suggesting that OSA may be a risk factor for hypertension (HTN) [4], coronary heart disease [5], cardiac arrhythmias [6], heart failure [7], stroke [8], and sudden death [9]. Although the specific mechanisms to explain the individual associations (noted above) have not been fully demonstrated, plausible mechanisms that contribute to an overall increased vascular risk in the setting of underlying OSA have 
been investigated. They include (but are not limited to) sympathetic activation [10], metabolic dysregulation [11], endothelial dysfunction [12], and oxidative stress [13].

Several studies $[14,15]$ have also focused on sex differences in OSA and examined the mechanisms as they relate to the underlying pathophysiology and clinical presentation of OSA. These include hormonal factors - specifically estrogen, testosterone and leptin [16], upper airway dimensions [17], craniofacial anatomic differences [18], and physiological response to fat and fluid distribution [19], particularly in the upper body which seems to be critical in the pathophysiology of OSA in men. There are also differences in the clinical presentation of OSA that likely contribute to increased prevalence of OSA in men compared to women. Women are less likely to report typical OSA symptoms such as snoring, witnessed apneas and excessive daytime sleepiness that are often used in prediction tools for OSA, rather they are more likely to present with symptoms such as insomnia, depression, and lack of energy. These symptoms are not typically included in prediction tools for OSA [20]. As a result, over $90 \%$ of women with moderate to severe OSA are often not diagnosed [21].

Although sex-related differences in the epidemiology and clinical presentation of OSA have been previously examined and reported, reports or reviews of sex differences in OSA as it relates to cardiovascular outcomes are not abundant. We therefore conducted a systematic review of the most recent medical literature to provide a more precise estimate of the effect of sex on the OSAcardiovascular outcome association. We discuss HTN, coronary heart disease, stroke, cardiac arrhythmias, and cardiovascular mortality as our main cardiovascular outcomes of interest in this review paper.

\section{Search Strategy}

We included observational and randomized controlled studies that satisfied the following inclusion criteria: (1) Study population was diagnosed with OSA, with or without reported polysomnography data; (2) age $\geq$ 18 years; (3) cardiovascular disorders such as HTN, coronary heart disease, stroke, cardiac arrhythmias, and mortality were reported; and (4) an English language version of the study was available.

A comprehensive computer-based search of the published medical literature was performed using PubMed. Keywords included in our search were sleep apnea, obstructive sleep apnea, OSA, coronary heart disease, cardiovascular disease, sex differences, cardiovascular outcomes, coronary artery disease, heart failure, chronic heart failure, sex differences, HTN, and stroke. We reviewed studies that were published between 1999 and 2015.

\section{Selection Process}

The first author $(\mathrm{ZN})$ reviewed eligible articles using the title and abstract. If an article was thought to be potentially eligible by ZN, the full-text article was subsequently reviewed. The reference lists of relevant review citations and references of all included citations were checked for additional potentially eligible studies.

\section{OSA and Hypertension}

OSA has been associated with HTN, and the evidence suggesting a causal association has been discussed in clinical reports since the mid-1980s. Various potential pathophysiologic mechanisms including endothelial dysfunction, sympathetic activation, and oxidative stress among others have been hypothesized and investigated. However, the role of sex on the relationship between OSA and HTN is less understood. In a cross-sectional study [22] of 1060 middle-aged men and women with polysomnography confirmed OSA, a linear increase in blood pressure with increasing AHI was demonstrated independent of confounding factors $(p=0.003$ for systolic and $p=0.01$ for diastolic adjusted for confounding factors). Subjects with an $\mathrm{AHI} \geq 15$ were more likely to be diagnosed with HTN (OR 1.8, 95 \% CI 1.3-2.4). However, sex did not modify this relationship and a non-significant interaction between AHI and sex was reported.

Nieto et al. [23] reported a cross-sectional analysis of participants in the Sleep Heart Health Study $(n=6132$, age $\geq 40$, $52.8 \%$ females) who underwent an unattended home polysomnogram. After adjusting for BMI, smoking, alcohol intake, neck circumference and waist to hip ratio, an $\mathrm{AHI} \geq 30$ was significantly associated with HTN $(\mathrm{OR}=1.37,95 \% \mathrm{CI}$ $1.03-1.83, p=0.005)$. Further, in a sex-stratified analysis with adjustment for confounding variables noted above, severe sleep apnea $(\mathrm{AHI} \geq 30)$ was associated with $\mathrm{HTN}$ among men ( $n=2897$; OR 1.49, $95 \%$ CI 1.06-2.12) but not among women $(n=3235$; OR $1.17,95 \%$ CI $0.74-1.86)$. In another analyses [24] of the longitudinal data collected in the Sleep Heart Health Study, 2740 participants over the age of 40, without HTN at baseline were followed in a prospective manner for the manifestation of incident HTN (defined as blood pressure of 140/90 mmHg or taking antihypertensive medication). When the data was stratified by sex, there was a significant association between severe OSA (AH I $\geq 30)$ and incident HTN in women but not in men (men OR 1.10, $95 \% \mathrm{CI}$ 0.57-2.10; women OR 2.27, $95 \%$ CI 1.07-4.80). The overall association between $\mathrm{OSA}(\mathrm{AHI} \geq 30)$ and incident HTN over 5 years was not statistically significant after adjusting for BMI (OR 1.51; $95 \%$ CI, 0.93-2.47). This study has to be interpreted with caution as it included a very small sample size of women with severe OSA, and the authors point out that this relationship still needs to be investigated further. 
Another large $(\mathrm{men}=741$; women $=1000)$ populationbased study by Bixler et al. [25] confirmed an independent association between OSA (AHI $\geq 15)$ and HTN (OR 1.72, $95 \%$ CI 1.24-2.55). However, sex did not influence this association between HTN and OSA. Contradicting findings have been reported by other clinic-based studies. While some report a higher likelihood of HTN in females [26], others report a significant influence of male sex on the association between HTN and OSA [27].

In summary, despite extensive epidemiological evidence of the relationship between HTN and OSA, there are inconsistencies on the influence of sex as an effect modifier of this association. This is partly due to varying study designs, selection bias, and inadequate power. Future prospective population-based cohort studies with adequate representation of men and women and with a wide range of OSA severity are needed to address the above noted inconsistencies.

\section{OSA and Coronary Artery Disease}

OSA is common among patients with underlying coronary artery disease (CAD) [28, 29]. Multiple observational studies have described OSA as an independent predictor of CAD [7]. Using angiography to objectively verify CAD, Schafer et al. [30] described a significant association between CAD and moderately severe OSA (OR 2.0; $95 \%$ CI, 1.0-3.8; $p<0.05)$; however, women were not included in the study sample. Since women were either excluded from most other studies or risk stratification based on sex was not performed, an inference on sex-related differences could not be determined.

A longitudinal analysis of participants from the Sleep Heart Health Study reported no significant association between OSA severity and incident CAD events among women [31]. When only considering men with severe OSA (AHI>30), adjusted for age, race, BMI, and smoking status, there was a weak yet statistically significant association with incident CAD (HR 1.45, $95 \%$ CI 0.99-2.13, $p=0.046$ ). Another recent analysis reported on the Wisconsin Sleep Cohort of 1280 participants (ages 30-60 years) who had a baseline overnight polysomnography and had follow-up data on self-reported CAD outcomes for up to 24 years [32••]. In this prospective population-based cohort study, they found that OSA was an independent risk factor for the development of the composite endpoint of CAD or heart failure (HR of 2.6 [1.1-6.1] for $\mathrm{AHI}>30$ compared to $\mathrm{AHI}=0$ ). Further analysis did not find sex to be a significant effect modifier of the development of CAD or heart failure in patients with OSA $(p=0.18)$.

In contrast to the association between OSA and HTN, emerging data have failed to show a robust association between OSA and CAD [31] (alone, not combining with other endpoints such as stroke or heart failure). This is intriguing and suggests that further work is needed to better understand whether the strength of the association between OSA and cardiovascular disease varies by specific outcome. For example, is OSA a stronger risk factor for HTN than it is for CAD? This remains to be determined definitively in prospective studies. Pertinent to this review, there is not enough longitudinal data to suggest that sex influences the OSA and CAD association.

\section{OSA and Stroke}

OSA has also been reported as an independent risk factor for stroke [33]. The fluctuations in blood pressure, reduction in cerebral blood flow, altered cerebral auto-regulation, endothelial dysfunction, accelerated atherogenesis, and prothrombotic states are some of the mechanisms that have been implicated in the increased risk of stroke in OSA patients [34].

Some studies support a stronger association of OSA and stroke in men compared to women [35]. Using the data from the Sleep Heart Health Study, Redline et al. aimed to quantify the incidence of stroke in a community-based multicenter prospective cohort study and provided sex-stratified data. In men, each AHI quartile increase was found to be associated with increased risk of incident ischemic stroke $(p<0.005)$, even after adjustment for confounders such as BMI, race, smoking, systolic blood pressure, antihypertensive medication, and diabetes. In women, stroke risk was only associated with severe OSA (AHI $\geq 25)$, but not at the lower levels of AHI severity which was in contrast to what was observed in men. There was no clear explanation for this observation, and the authors concluded that more studies of women with severe OSA are needed to make definitive conclusions about their findings.

Campos et al. [36・•] accomplished this task by conducting a prospective observational study in women only. They found that untreated OSA $(\mathrm{AHI} \geq 10)$ was associated with an increased incidence of serious cardiovascular outcomes, particularly stroke (adjusted HR 6.44, 95 \% CI 1.46-28.3). Notably, this study also found that adequate treatment with continuous positive airway pressure (CPAP) could reduce the risk of the composite cardiovascular outcome (HR of $2.76,95 \% \mathrm{CI}$ 1.35-5.62, for the untreated OSA group and HR 0.91, $95 \%$ CI $0.43-1.95$, for the CPAP treated group). In contrast to the study noted earlier, the strength of this study was that it included higher average severity of OSA. However, since it was limited to women only, sex-stratified analysis could not be performed.

A recent retrospective observational cohort study [37••] conducted in Taiwan compared sleep apnea syndrome (SAS) cohort $(n=29,961)$ to a non-SAS cohort $(n=119,844)$ found the overall incidence of stroke in the SAS cohort was $37 \%$ higher compared to the non-SAS cohort (54.6 per 10,000 individual years vs. 39.8 per 10,000 individual years). Contrary to the Campos et al. study, sex-stratified analysis was performed. After controlling for sex and comorbidities, the SAS 
cohort exhibited a $19 \%$ higher stroke risk compared to the control cohort (adjusted HR 1.19, 95 \% CI 1.09-1.30). Additionally, the authors also reported that the risk of stroke associated with SAS among women was higher compared to their male counterparts (with adjusted HRs in men and women of 1.21 [95\% CI, 1.01-1.24; $p<0.05$ ] and 1.44 [95\% CI, $1.20-1.72 ; p<0.05]$, respectively).

To further investigate the relationship between OSA and stroke, Dyken et al. [38] involved 24 patients with stroke and 27 patients without stroke and explored the sex differences in the prevalence and severity of OSA. The severity of OSA was much higher among the women in the group (mean AHI 31.6 \pm 8.8 events per hour) when compared with men (mean AHI $21.5 \pm 4.2$ events per hour).

OSA is being increasingly recognized as a risk factor for stroke in women especially among those with higher severity of OSA. Future work is needed to explain differences in the OSA and stroke association by sex as well as the impact of OSA treatment on stroke outcomes by sex.

\section{OSA and Cardiac Arrhythmias}

OSA has been studied for three decades as a potential target for therapy to prevent arrhythmias. A strong association with cardiac arrhythmias has been reported [6, 39]; however, the pathophysiology behind the causality of these arrhythmias is still under investigation. Proposed mechanisms for arrhythmogenic properties of OSA include acute hypoxemia, hypercapnea, surges in sympathetic nerve system activity, increased myocardial oxygen demand, and increased cardiac work load $[40 \bullet \bullet$.

OSA was found to be independently associated with atrial fibrillation (OR 4.02; $95 \%$ CI, 1.03-15.74), non-sustained ventricular tachycardia (OR 3.40; $95 \% \mathrm{CI}, 1.03-11.20$ ), and complex ventricular ectopy (OR 1.74; $95 \% \mathrm{CI}, 1.11-2.74$ ), after adjustment for confounding variables among participants of the Sleep Heart Health Study [6]. Another study [41] reported a higher prevalence of atrial fibrillation in patients with OSA (OR 1.89, $95 \%$ CI 1.40-2.83, $p=0.002$ ) and found OSA was a strong predictor of incident atrial fibrillation (HR 2.18, $95 \%$ CO 1.34-3.54, $p=0.002$ ). However, sex-stratified analysis was not performed in these studies.

Expanding on the study by Gami et al. [41] Cadby et al. [42.0. looked at incidence of atrial fibrillation in patients with OSA in a larger cohort with a longer follow-up time. The study included 6841 patients who had an in-laboratory sleep study. While confirming that men were more likely to have incident atrial fibrillation (HR 1.32, $95 \%$ CI 1.04-1.68, $p=$ 0.022 ), they reported that multivariate analysis did not show effect modification by sex on the relationship of AHI severity and incidence of atrial fibrillation $(p>0.05)$; however, more detailed data was not provided.
Another cross-sectional population study by Cintra et al. [43••] looked at incidence of nocturnal arrhythmias in patients with OSA using in-laboratory sleep studies. Cardiac rhythm disturbance was observed in $53.3 \%$ of subjects without sleep breathing disorders, while $92.3 \%$ of patients with severe OSA $(\mathrm{AHI} \geq 15)$ had cardiac arrhythmias. After controlling for potential confounders (age, BMI, smoking, diabetes, HTN, and polysomnography parameters), male sex was independently associated with the occurrence of nocturnal cardiac arrhythmia (HR 1.49, $95 \%$ CI 1.04-2.16, $p=0.032$ ); however, statistical testing for the effect modification by sex on the OSA and nocturnal cardiac arrhythmias was not reported in this study.

In summary, there is persuasive evidence to support an association between OSA and cardiac arrhythmias especially atrial fibrillation. However, the studies highlighted above do not provide a consensus on how sex modifies the OSA and cardiac arrhythmias relationship.

\section{OSA and Cardiovascular Mortality}

The association between OSA and mortality has been well characterized by prior studies; however, most lack data on cardiovascular mortality specifically, few studies include women, and even fewer have sex-stratified data. For example, in a prospective observational cohort study by Campos et al. [44] including women only, severe untreated OSA (AHI $\geq 30)$ was independently associated with cardiovascular mortality (HR 3.50, $95 \%$ CI 1.23-9.98, $p=0.019$ ). With adequate CPAP treatment, mortality in the severe OSA group did not differ from the control group (HR $0.55,95 \%$ CI 0.17 to 1.74 , $p=0.31$ ). Although this study was limited to women, it is important to include it here because most other studies were conducted primarily in men and these findings differ substantially from the Sleep Heart Health Study analysis discussed further on.

In analysis of the Sleep Heart Health Study [45] (6294 participants, $53.3 \%$ women), a total of 1047 participants being followed for 8.2 years had died at follow-up, of which 587 were men and 460 were women. In an exploratory analysis, they report an effect modification by sex; men $\leq 70$ with severe OSA (AHI $\geq$ 30) had an increased risk of all-cause mortality (HR $2.09,1.31$ to 3.33 ) after adjusting for confounding variables. Women and men older than 70 years of age did not show a similar association. The difference between the findings in the above two studies could be accounted for by the fact that the Sleep Heart Health Study had a lower percentage of women with severe OSA $(\mathrm{AHI} \geq 25)$ and had a different endpoint of allcause mortality.

Korostovtseva et al. [46] studied OSA patients with newly diagnosed or uncontrolled HTN to assess the impact of OSA 
on cardiovascular morbidity and mortality. They found that severe OSA $(\mathrm{AHI} \geq 30)$ was associated with a higher risk of cardiovascular events such as cardiovascular death and fatal and non-fatal myocardial infarction $(\mathrm{OR}=8.557,95 \% \mathrm{CI}$ $1.142-64.131, p=0.037)$. In this study, the relationship of OSA and mortality was not modified by sex.

A more recent study evaluated all-cause mortality from OSA in over 25,000 middle-aged and elderly males and females with OSA who were treated or not treated with CPAP therapy. They found that female OSA patients had a lower mortality than males (regardless of OSA treatment status) $[47 \bullet \bullet]$.

There appears to be limited evidence to examine whether sex is an effect modifier of the relationship between OSA and cardiovascular mortality. The vast majority of the studies examining mortality in OSA have either assessed all-cause mortality only as an endpoint without further validation of cause-specific mortality, and when they have ascertained cardiovascular mortality, they did not assess sex as an effect modifier of the cardiovascular disease mortality endpoint. Therefore, definitive conclusions cannot be drawn using existing evidence on whether the association between OSA and cardiovascular disease mortality varies by sex.

\section{Conclusion}

We chose to focus on five major cardiovascular endpoints associated with OSA, i.e., HTN, coronary heart disease, stroke, arrhythmias, and death. Even though there is accruing evidence to suggest that the association between OSA and cardiovascular diseases is robust, most studies have inadequately addressed the question of whether sex influences this relationship. The studies that did assess sex as an effect modifier (including the ones we listed above) have done so without adequate power and/or as secondary/exploratory analyses. As such, this limits our ability to make any definitive conclusions. Further investigation is still warranted to discern the effect of sex on the relationship between OSA and cardiovascular disease.

Acknowledgments NS received grant funding from Albert Einstein College of Medicine's ICTR-CTSA K12 grant (award period 7/1/20126/30/2015).

\section{Compliance with Ethics Guidelines}

Conflict of Interest Zarina Nestor, Sapna Siddharth, Ridhwan Y. Baba, and Neomi Shah declare that they have no conflict of interest.

Human and Animal Rights and Informed Consent This article does not contain any studies with human or animal subjects performed by any of the authors.

\section{References}

Papers of particular interest, published recently, have been highlighted as:

-• Of major importance

1.• Peppard PE, Young T, Barnet JH, et al. Increased prevalence of sleep-disordered breathing in adults. Am J Epidemiol. 2013;177(9):1006-14. The present analysis necessarily updates and substantially extends earlier Wisconsin Sleep Cohort Study estimates of the prevalence of sleep disordered breathing.

2. Redline S, Strohl KP. Recognition and consequences of obstructive sleep apnea hypopnea syndrome. Otolaryngol Clin N Am. 1999;32(2):303-31.

3. Redline S, Kump K, Tishler PV, et al. Gender differences in sleep disordered breathing in a community-based sample. Am J Respir Crit Care Med. 1994;149(3 Pt 1):722-6.

4. Peppard P, Young T, Palta M, et al. Prospective study of the association between sleep-disordered breathing and hypertension. NEJM. 2000;342(19):1378-84.

5. Peker Y, Carlson J, Hedner J. Increased incidence of coronary artery disease in sleep apnoea: a long-term follow-up. Eur Respir J. 2006;28(3):596-602.

6. Mehra R, Benjamin EJ, Shahar E, et al. Association of nocturnal arrhythmias with sleep-disordered breathing: the Sleep Heart Health Study. Am J Respir Crit Care Med. 2006;173(8):910-6.

7. Shahar E, Whitney CW, Redline S, et al. Sleep-disordered breathing and cardiovascular disease: cross-sectional results of the Sleep Heart Health Study. Am J Respir Crit Care Med. 2001;163(1):1925.

8. Yaggi H, Brass L, Kernan W, et al. Obstructive sleep apnea as a risk factor for stroke. Stroke. 2004;316:365.

9. Gami AS, Howard DE, Olson EJ, et al. Day-night pattern of sudden death in obstructive sleep apnea. N Engl J Med. 2005;352(12): 1206-14.

10. Somers V, Dyken M, Clary M, et al. Sympathetic neural mechanisms in obstructive sleep apnea. J Clin Invest. 1995;96:1897-904.

11. Drager LF, Jun JC, Polotsky VY. Metabolic consequences of intermittent hypoxia: relevance to obstructive sleep apnea. Best Pract Res Clin Endocrinol Metab. 2010;24(5):843-51.

12. Jelic S, Padeletti M, Kawut SM, et al. Inflammation, oxidative stress, and repair capacity of the vascular endothelium in obstructive sleep apnea. Circulation. 2008;117(17):2270-8.

13. Yamauchi M, Nakano H, Maekawa J, et al. Oxidative stress in obstructive sleep apnea. Chest. 2005;127(5):1674-9.

14. Drager LF, Togeiro SM, Polotsky VY, et al. Obstructive sleep apnea: a cardiometabolic risk in obesity and metabolic syndrome. $\mathrm{J}$ Am Coll Cardiol. 2013.

15. Shamsuzzaman AS, Gersh BJ, Somers VK. Obstructive sleep apnea: implications for cardiac and vascular disease. JAMA. 2003;290(14):1906-14.

16. Saaresranta T, Polo O. Sleep-disordered breathing and hormones. Eur Respir J. 2003;22(1):161-72.

17. Mohsenin V. Effects of gender on upper airway collapsibility and severity of obstructive sleep apnea. Sleep Med. 2003;4(6):523-9.

18. Guilleminault C, Quera-Salva MA, Partinen M, et al. Women and the obstructive sleep apnea syndrome. Chest. 1988;93(1):104-9.

19. Millman RP, Carlisle CC, McGarvey ST, et al. Body fat distribution and sleep apnea severity in women. Chest. 1995;107(2):362-6.

20. O'Connor C, Thornley KS, Hanly PJ. Gender differences in the polysomnographic features of obstructive sleep apnea. Am J Respir Crit Care Med. 2000;161(5):1465-72. 
21. Young T, Evans L, Finn L, et al. Estimation of the clinically diagnosed proportion of sleep apnea syndrome in middle-aged men and women. Sleep. 1997;20(9):705-6.

22. Young T, Peppard P, Palta M, et al. Population-based study of sleepdisordered breathing as a risk factor for hypertension. Arch Intern Med. 1997;157(15):1746-52.

23. Nieto FJ, Young TB, Lind BK, et al. Association of sleepdisordered breathing, sleep apnea, and hypertension in a large community-based study. Sleep Heart Health Study. JAMA. 2000;283(14):1829-36.

24. O'Connor GT, Caffo B, Newman AB, et al. Prospective study of sleep-disordered breathing and hypertension: the Sleep Heart Health Study. Am J Respir Crit Care Med. 2009;179(12):1159-64.

25. Bixler EO, Vgontzas AN, Lin HM, et al. Association of hypertension and sleep-disordered breathing. Arch Intern Med. 2000;160(15):2289-95.

26. Drager LF, Pereira AC, Barreto-Filho JA, et al. Phenotypic characteristics associated with hypertension in patients with obstructive sleep apnea. J Hum Hypertens. 2006;20(7):523-8.

27. Mohsenin V, Yaggi HK, Shah N, et al. The effect of gender on the prevalence of hypertension in obstructive sleep apnea. Sleep Med. 2009; 10(7):759-62.

28. Mooe T, Rabben T, Wiklund U, et al. Sleep-disordered breathing in women: occurrence and association with coronary artery disease. Am J Med. 1996;101(3):251-6.

29. Mooe T, Rabben T, Wiklund U, et al. Sleep-disordered breathing in men with coronary artery disease. Chest. 1996;109(3):659-63.

30. Schafer H, Ewig S, Hasper E, et al. Sleep apnea as a risk marker in coronary heart disease. Z Kardiol. 1996;85(10):768-75.

31. Gottlieb DJ, Yenokyan G, Newman AB, et al. Prospective study of obstructive sleep apnea and incident coronary heart disease and heart failure: the Sleep Heart Health Study. Circulation. 2010;122(4):352-60.

32.• Hla KM, Young T, Hagen EW, et al. Coronary heart disease incidence in sleep disordered breathing: the Wisconsin Sleep Cohort Study. Sleep. 2015;38(5):677-84. Study which supports the postulated adverse effects of sleep disordered breathing on coronary artery disease and heart failure and also finds a stronger association compared to the sleep heart health study.

33. Yaggi HK, Concato J, Kernan WN, et al. Obstructive sleep apnea as a risk factor for stroke and death. N Engl J Med. 2005;353(19): $2034-41$.

34. Somers VK, White DP, Amin R, Abraham WT, Costa F, Culebras A, et al. Sleep apnea and cardiovascular disease: an American Heart Association/American College of Cardiology Foundation Scientific Statement from the American Heart Association Council for High Blood Pressure Research Professional Education Committee, Council on Clinical Cardiology, Stroke Council, and Council on Cardiovascular Nursing. J Am Coll Cardiol. 2008;52:686-717.

35. Redline S, Yenokyan G, Gottlieb DJ, et al. Obstructive sleep apneahypopnea and incident stroke: the Sleep Heart Health Study. Am J Respir Crit Care Med. 2010;182(2):269-77.

36.• Campos-Rodriguez F, Martinez-Garcia MA, Reyes-Nunez N, et al. Role of sleep apnea and continuous positive airway pressure therapy in the incidence of stroke or coronary heart disease in women. Am J Respir Crit Care Med. 2014;189(12):1544-50. Study which specifically investigates the role of OSA and positive pressure therapy on the incidence of stroke or coronary heart disease in women.

37.• Chang CC, Chuang HC, Lin CL, et al. High incidence of stroke in young women with sleep apnea syndrome. Sleep Med. 2014;15(4): 410-4. Addresses differential stroke risk between the sexes and among different age group which has not been done in previous studies.

38. Dyken M, Somers V, Yamada T, et al. Investigating the relationship between stroke and obstructive sleep apnea. Stroke. 1996;27:4017.

39. Mehra R, Stone KL, Varosy PD, et al. Nocturnal arrhythmias across a spectrum of obstructive and central sleep-disordered breathing in older men: outcomes of sleep disorders in older men (MrOS sleep) study. Arch Intern Med. 2009;169(12):1147-55.

$40 . \bullet$ Bitter T, Fox H, Gaddam S, et al. Sleep-disordered breathing and cardiac arrhythmias. Can J Cardiol. 2015;31(7):928-34. An important review on the most up to date findings regarding OSA and its arrythmogenesis, including current treatment options.

41. Gami AS, Pressman G, Caples SM, et al. Association of atrial fibrillation and obstructive sleep apnea. Circulation. 2004;110(4): 364-7.

42.• Cadby G, McArdle N, Briffa T, et al. Severity of obstructive sleep apnea is an independent predictor of incident atrial fibrillation hospitalization in a large sleep-clinic cohort. Chest. 2015. To date, one of the largest clinic-based cohort studies of the association of OSA and incident AF with a long 12-year median follow-up.

43.• Cintra FD, Leite RP, Storti LJ, et al. Sleep apnea and nocturnal cardiac arrhythmia: a populational study. Arq Bras Cardiol. 2014;103(5):368-74. The result of this study demonstrated that the prevalence of nocturnal cardiac arrhythmia was higher than that previously described in the literature.

44. Campos-Rodriguez F, Martinez-Garcia MA, de la Cruz-Moron I, et al. Cardiovascular mortality in women with obstructive sleep apnea with or without continuous positive airway pressure treatment: a cohort study. Ann Intern Med. 2012;156(2):115-22.

45. Punjabi NM, Caffo BS, Goodwin JL, et al. Sleep-disordered breathing and mortality: a prospective cohort study. PLoS Med. 2009;6(8):e1000132.

46. Korostovtseva LS, Sviryaev YV, Zvartau NE, et al. Prognosis and cardiovascular morbidity and mortality in prospective study of hypertensive patients with obstructive sleep apnea syndrome in St Petersburg, Russia. Med Sci Monit. 2011;17(3):CR146-53.

47.• Jennum P, Tonnesen P, Ibsen R, et al. All-cause mortality from obstructive sleep apnea in male and female patients with and without continuous positive airway pressure treatment: a registry study with 10 years of follow-up. Nat Sci Sleep. 2015;7:43-50. Study which finds female patients with OSA having lower mortality compared to males suggesting a possible protective effect of gender on the consequences of OSA which needs to be explored in further studies. 